\title{
Efficient Message Logging for Uncoordinated Checkpointing Protocols
}

Achour Mostefaoui , Michel Raynal

$$
N^{\circ} 2972
$$

Septembre 1996

THÈME 1 



\title{
Efficient Message Logging for Uncoordinated Checkpointing Protocols
}

\author{
Achour Mostefaoui, Michel Raynal \\ Thème 1 - Réseaux et systèmes \\ Projet Adp
}

Rapport de recherche $\mathrm{n}^{\circ} 2972$ - Septembre 1996 - 11 pages

\begin{abstract}
A message is in-transit with respect to a global state if its sending is recorded in this global state, while its receipt is not. Checkpointing algorithms have to log such in-transit messages in order to restore the state of channels when a computation has to be resumed from a consistent global state after a failure has occurred. Coordinated checkpointing algorithms log those in-transit messages exactly on stable storage. Because of their lack of synchronization, uncoordinated checkpointing algorithms conservatively log more messages.

This paper presents an uncoordinated checkpointing protocol that logs all in-transit messages and the smallest possible number of non in-transit messages. As a consequence, the protocol saves stable storage space and enables quicker recoveries. An appropriate tracking of message causal dependencies constitutes the core of the protocol.
\end{abstract}

Key-words: Distributed Systems, Backward Recovery, Consistent Global Checkpoints, Optimistic Sender-Based Logging

(Résumé : tsvp)

IRISA, Campus de Beaulieu, Rennes Cedex, France. Email: \{mostefaoui,raynal\}irisa.fr

To appear in the Proceedings of EDCC2 (2nd European Dependable Computing Conference), october 1996. 


\section{Enregistrement sélectif de messages lors d'une définition non coordonnée de points de contrôle}

Résumé : Les algorithmes de définition de points de reprise nécessitent l'enregistrement de certains messages dits en transit afin de pouvoir restaurer l'état des canaux lors de recouvrements arrières. Un message est en transit si son émission est enregistrée dans l' état global de l'application alors que sa réception de l'est pas. Les algorithmes coordonnés enregistrent exactement en mémoire stable les messages en transit relativement a l'état global calculé alors que les algorithmes non coordonnés enregistrent la totalité des messages. Cet article, d'une part, définit une classe de messages qui ne peuvent être en transit dans aucun état global cohérent et d'autre part, propose un algorithme qui n'enregistre pas ce type de messages et permet ainsi de réduire l'occupation de la mémoire stable.

Mots-clé : Enregistrement optimiste de messages, états globaux cohérents, points de reprise, recouvrement arrière 


\section{Introduction}

Checkpointing and backward recovery are well-known techniques for providing fault-tolerance in asynchronous systems. During the execution of an application a checkpointing algorithm computes and saves global checkpoints (global states) of the computation on stable storage. When a fault occurs, a backward recovery algorithm restores the computation in the most up to date global checkpoint from which this computation can be resumed.

A global checkpoint is composed of two parts: (1) a local checkpoint (local state) for each application process, and (2) a set of messages (channel state) for each communication channel. Informally, a global checkpoint is consistent if the computation could have passed through it.

A lot of checkpointing algorithms have been defined. They can be divided into two classes according to the way local checkpoints are determined. In the class of coordinated algorithms [5, 9, 17], the determination of local checkpoints and the computation of corresponding channel states are synchronized in such a way that the resulting global checkpoints are always consistent.

The main disadvantage of this approach is the added synchronization requiring control messages that can slow down or even freeze the computation for a given duration [9]. Its main advantage is that the last global checkpoint computed (as it is consistent) is the only one that has to be kept in stable storage. Moreover, when a fault occurs, the only work of a backward recovery algorithm consists in installing this global checkpoint and resuming the computation from it.

In the class of uncoordinated checkpointing algorithms $[2,6,7,8,19,21]$, processes save local checkpoints on stable storage in an independent way. Furthermore, they also log messages on stable storage so that channel states can be determined. When a failure occurs, it is up to the backward recovery algorithm to compute a consistent global state from local checkpoints [18] and messages saved on stable storage. The message logging technique is called sender-based (respt. receiver-based) if messages are logged by their senders (respt. receivers). Two logging techniques are possible. In the case of pessimistic logging, messages are logged on stable storage at the time they are sent (respt. received). This can incur high overhead in failure-free executions as each sending (respt. receiving) entails an additional input/output. This is why optimistic message logging techniques have been developed. In that case, when a message is sent (respt. received), it is saved on a volatile log and this $\log$ is saved on stable storage when the process takes a local checkpoint (as all messages are saved, the stable storage contains all in-transit messages).

In this paper we are interested in uncoordinated checkpointing algorithms with optimistic senderbased message logging. We develop a technique allowing a process to save the smallest possible number of volatile log messages on stable storage. This subset is consistent in the sense that it includes all the messages which are in-transit with respect to any consistent global checkpoint from which the computation could be resumed.

This paper is composed of two main parts. Section 2 introduces the model of distributed computations and formally defines the consistency of a global checkpoint. Section 3 presents the checkpointing algorithm.

\section{Consistent Global Checkpoints}

\subsection{Distributed Computations}

A distributed program is made up of $n$ sequential local programs which, when executed, can communicate and synchronize only by exchanging messages. A distributed computation describes the execution of a distributed program. The execution of a local program gives rise to a sequential process. Let $P_{1}, P_{2}, \ldots, P_{n}$ be this finite set of processes. We assume that, at run-time, each ordered pair of communicating processes $\left(P_{i}, P_{j}\right)$ is connected by a reliable and FIFO channel $c_{i j}$ through which $P_{i}$ can send messages to $P_{j}$. Message transmission delays are finite but unpredictable. Process speeds are

RR n ${ }^{\circ} 2972$ 


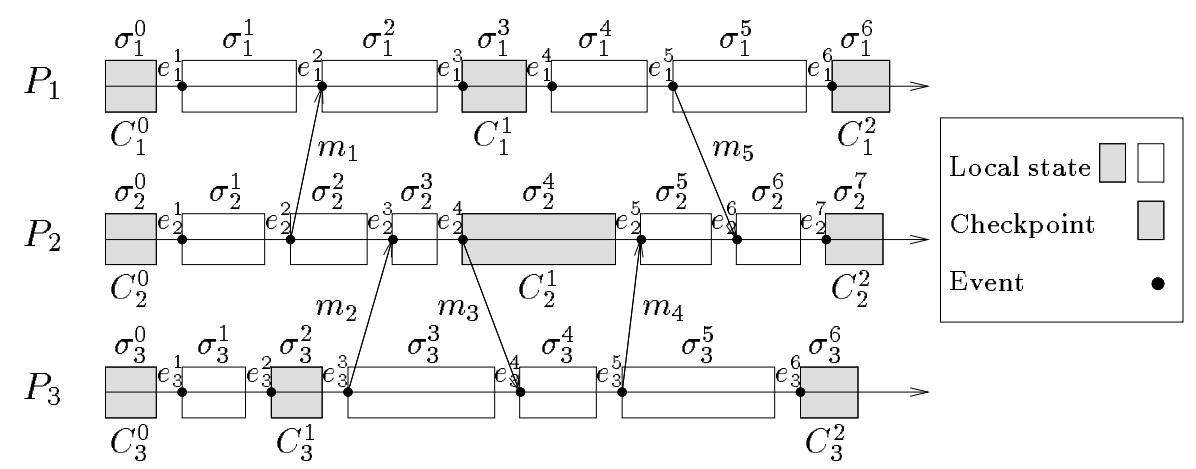

Figure 1: Local states of a distributed computation.

positive but arbitrary. In other words, the underlying computation model is asynchronous. Processes can fail by crashing, i.e. halting prematurely. The local program associated with $P_{i}$ can include send, receive and internal statements; some statements can be non-deterministic ${ }^{1}$.

Execution of an internal, send or receive statement produces an internal, send or receive event. Let $e_{i}^{x}$ be the $x^{t h}$ event produced by process $P_{i}$. The sequence $h_{i}=e_{i}^{1} e_{i}^{2} \ldots e_{i}^{x} \ldots$ constitutes the history of $P_{i} . h_{i}^{x}$ denotes the partial history $e_{i}^{1} \ldots e_{i}^{x}$ of process $P_{i}$.

Let $H$ be the set of events produced by a distributed computation. This set is structured as a partial order by Lamport's causal precedence relation [10], denoted " $\rightarrow$ " and defined as follows:

$e_{i}^{x} \rightarrow e_{j}^{y}$ if, and only if:

1. $i=j$ and $x \leq y$, or

2. $e_{i}^{x}$ is the sending of a message whose receiving is $e_{j}^{y}$, or

3. $\exists e_{k}^{z}: e_{i}^{x} \rightarrow e_{k}^{z}$ and $e_{k}^{z} \rightarrow e_{j}^{y}$ (transitive closure).

The partial order $\widehat{H}=(H, \rightarrow)$ constitutes a model of the distributed execution it is associated with.

\subsection{Local States and Local Checkpoints}

Let $\sigma_{i}^{0}$ be the initial state of process $P_{i}$. Event $e_{i}^{x}$ entails $P_{i}$ 's local state change from $\sigma_{i}^{x-1}$ to $\sigma_{i}^{x}$ : $\sigma_{i}^{x}$ is the local state of $P_{i}$ resulting from its local history $h_{i}^{x}$; we say that $e_{i}^{y}$ belongs to $\sigma_{i}^{x}$ if $y \leq x$. Figure 1 depicts a distributed computation where events are denoted by black points and local states by (white or grey) rectangular boxes.

Let $\Sigma$ be the set of all local states associated with a distributed computation $\hat{H}$. Lamport's precedence relation can be extended to local states in the following way:

$$
\sigma_{i}^{x} \rightarrow \sigma_{j}^{y} \Leftrightarrow e_{i}^{x+1} \rightarrow e_{j}^{y}
$$

Local states not related by " $\rightarrow$ " are said to be concurrent, (denoted $\|$ ); more formally:

$$
\sigma_{i}^{x} \| \sigma_{j}^{y} \Leftrightarrow \neg\left(\sigma_{i}^{x} \rightarrow \sigma_{j}^{y}\right) \text { and } \neg\left(\sigma_{j}^{y} \rightarrow \sigma_{i}^{x}\right)
$$

In Figure 1, we have $\sigma_{3}^{2} \rightarrow \sigma_{2}^{4}$ and $\sigma_{1}^{3} \| \sigma_{3}^{6}$.

A local checkpoint of process $P_{i}$ is a local state of $P_{i}$ saved on stable storage. So, the set of all local checkpoints is thus a subset of $\Sigma$. $C_{i}^{t}$ will denote the $t$-th local checkpoint of $P_{i}$; it corresponds

\footnotetext{
${ }^{1}$ When the only non-deterministic statements are "receive" statements, the program is piece-wise deterministic. If statements other than "receive" are non-deterministic the program is fully non-deterministic.
} 
to some local state $\sigma_{i}^{x}$ with $t \leq x$. We assume that each process takes an initial local checkpoint $C_{i}^{0}$ corresponding to $\sigma_{i}^{0}$. Grey rectangular boxes on Figure 1 depict local states that correspond to local checkpoints.

Determining which local states of a process are selected as local checkpoints, and consequently which are not, is the job of a checkpointing algorithm superimposed on the computation. When this algorithm defines the current local state of a process $P_{i}$ as a local checkpoint, it saves it on stable storage, and $P_{i}$ is said to "take a checkpoint".

\subsection{In-transit Messages}

A message $m$ is in-transit with respect to an ordered pair $\left(C_{i}^{s}, C_{j}^{t}\right)$ if its sending event belongs to $C_{i}^{s}$ while its receiving event does not belong to $C_{j}^{t}$. In Figure $1, m_{4}$ is in transit with respect to $\left(C_{3}^{2}, C_{2}^{1}\right)$. In-transit messages actually constitute channel states which have to be considered to define consistency of global checkpoints.

\subsection{Consistent Global Checkpoints}

Informally, a consistent global checkpoint is a global state of a computation through which this computation could have passed. It consists of: (1) a set of concurrent local checkpoints (one per process), and (2) for each channel $c_{i j}$, the sequence ${ }^{2}$ of messages that are in transit with respect to the ordered pair of local checkpoints of $P_{i}$ and $P_{j}$. Formally, let $C_{i}$ be a local checkpoint of process $P_{i}$ and $c_{-} s_{i j}$ a state of the channel $c_{i j}$, i.e. a sequence of messages sent by $P_{i}$ to $P_{j}$. A global checkpoint $G$ is defined as:

$$
G=\cup_{i}\left\{C_{i}\right\} \cup_{i, j}\left\{c_{-} s_{i j}\right\}
$$

$G$ is consistent if for any ordered pair $\left(P_{i}, P_{j}\right)$ :

- $\neg\left(C_{i} \rightarrow C_{j}\right)\left(C_{j}\right.$ does not depend on $\left.C_{i}\right)$.

- $c_{-} s_{i j}$ is the sequence of messages in transit with respect to $\left(C_{i}, C_{j}\right)$.

As an example, let us consider Figure 1. $C_{3}^{1}$ and $C_{2}^{1}$ cannot belong to a same consistent global checkpoint as $C_{3}^{1} \rightarrow C_{2}^{1}$. $C_{1}^{1}, C_{2}^{1}$ and $C_{3}^{2}$ can belong to a same consistent global checkpoint when considering all channel states empty except $c_{-} s_{32}=\left\{m_{4}\right\}$.

This consistency definition is the classic one used to define global checkpoints. The algorithms described in $[5,9]$ are classic examples of coordinated checkpointing algorithms. Due to the synchronization they use to define local checkpoints and associated channels states, coordinated checkpointing algorithms ensure the a priori consistency of the global checkpoints they determine.

\section{Efficient Optimistic Sender-Based Message Logging}

Uncoordinated checkpointing algorithms replace the previous a priori synchronization by tracking dependence on local checkpoints and by logging enough messages so that all in-transit messages can be retrieved to determine correct channel states. In the rest of this paper, we are interested in optimistic sender-based message logging.

\footnotetext{
${ }^{2}$ As we consider FIFO channels, their states are sequences of messages. If channels were not FIFO, their states would be sets of messages.
}

$\operatorname{RR} n^{\circ} 2972$ 


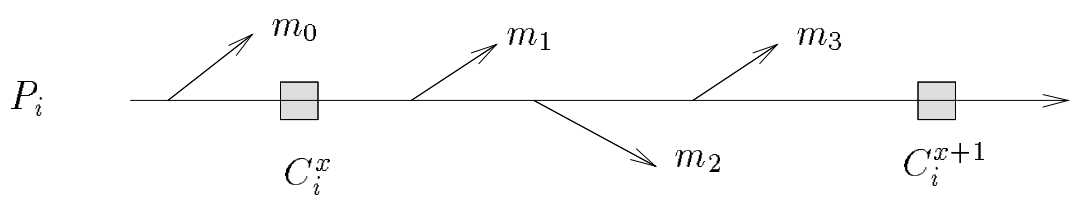

Figure 2: Sender-based message logging.

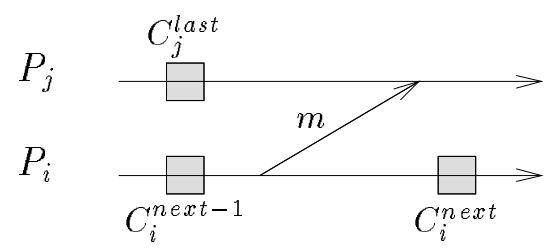

(a)

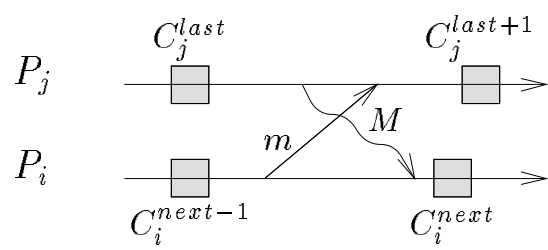

(b)

Figure 3: To $\log$ or not to $\log$.

\subsection{Optimistic Sender-Based Logging}

The principle of optimistic sender-based message logging is illustrated on Figure 2. When a message is sent, it is momentarily logged by its sender on a volatile log. More precisely, when $P_{i}$ takes a local checkpoint $C_{i}^{x},(1)$ it saves $C_{i}^{x}$ and the current content of the volatile log on the stable storage and (2) it re-initializes the volatile $\log$ to empty. Then $P_{i} \operatorname{logs}$ on its volatile $\log$ all the messages it sends till its next local checkpoint $C_{i}^{x+1}\left(m_{1}, m_{2}\right.$ and $m_{3}$ in the example depicted in Figure 2$)$. In this way, messages are saved on stable storage "by batch" and not independently. This decreases the volume of input/output and, consequently, the overhead associated with message logging.

\subsection{To Log or not to Log on Stable Storage?}

The question we want to answer is the following one: "given a message $m$ stored in its volatile log, should $P_{i} \log$ it on stable storage when it takes its next local checkpoint $C_{i}^{\text {next }}$ ?" Basically, a message has not to be saved on stable storage if it cannot be in transit in any consistent global checkpoint. To answer the previous question more precisely, let us consider the message $m$ in Figures 3.a and 3.b; $C_{j}^{\text {last }}$ denotes the last local checkpoint taken by $P_{j}$ before receiving $m$.

In Figure 3.a, $C_{j}^{\text {last }}$ and $C_{i}^{\text {next }}$ are concurrent and can therefore belong to a same consistent global checkpoint in which $c_{-} s_{i j}=\{m\}$. In this case, $m$, kept in the volatile log of $P_{i}$, must be saved on stable storage when $C_{i}^{\text {next }}$ is taken. In that way, this in-transit message can be retrieved if a consistent global checkpoint including $C_{j}^{\text {last }}$ and $C_{i}^{\text {next }}$ has to be restored.

Let us now consider Figure 3.b where $M$ denotes a causal chain ${ }^{3}$ of messages starting after $C_{j}^{\text {last }}$ and arriving at $P_{i}$ before $C_{i}^{\text {next }}$. We have then $C_{j}^{\text {last }} \rightarrow C_{i}^{\text {next }}$ from which we conclude that $C_{j}^{\text {last }}$ and $C_{i}^{\text {next }}$ cannot belong to the same consistent global checkpoint.

Let $C_{j}^{\text {last+1 }}$ be the first local checkpoint taken by $P_{j}$ after receiving $m$. When considering any global checkpoint including $C_{i}^{x}$ and $C_{j}^{y}$ :

- if $x \leq n e x t-1$ and $y \leq l a s t: m$ has been neither sent nor received.

- if $x \geq$ next and $y \geq$ last $+1: m$ has been sent and received.

\footnotetext{
${ }^{3}$ A causal chain of messages is characterized by the following property: every message of the chain (but the first one) is received, according to " $\rightarrow$ ", before the sending of the next one.
} 


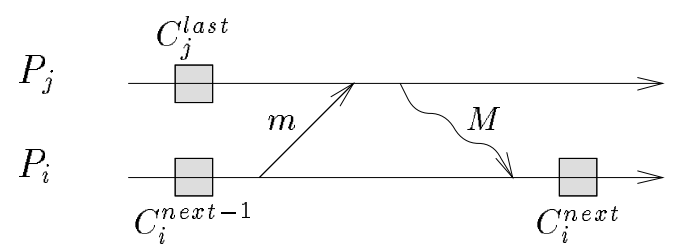

Figure 4: $P_{i}$ can decide not to log on stable storage.

As, additionally, $C_{j}^{\text {last }} \rightarrow C_{i}^{\text {next }}$ (because of $M$ ) and $C_{i}^{\text {next }-1} \rightarrow C_{j}^{\text {last }+1}$ (because of $m$ ), we can conclude that $m$ cannot be in transit in any consistent global checkpoint. It follows that $m$ need not be logged in stable storage when $P_{i}$ takes $C_{i}^{\text {next }}$.

Figure 3.b exhibits the pattern of local checkpoints and message exchanges in which a message $m$ has not to be logged on stable storage. The problem is now to answer the following question: "how can a process $P_{i}$ know that a message $m$ it sent to $P_{j}$ will never be in transit with respect to any pair of local checkpoints $\left(C_{i}^{s}, C_{j}^{t}\right)$ ?" The only way ${ }^{4}$ for $P_{i}$ to learn this is on receiving a message piggybacking the information " $m$ has been received by $P_{j}$ ". This situation is described in Figure 4 where the causal chain $M$ conveys this information to $P_{i}$. On the contrary, in Figure 3.b, the chain $M$ missed the information " $P_{j}$ receives $m$ ".

The uncoordinated checkpointing algorithm described in the next section exploits the flow of messages exchanged by a distributed computation to convey control information used to reduce the number of messages logged on stable storage by their senders.

\subsection{A Checkpointing Algorithm}

We assume an uncoordinated checkpointing algorith ${ }^{5}$ that uses an optimistic sender-based message logging strategy. We augment it, according to the previous discussion (Section 3.2), in order to reduce the number of messages transferred from a volatile log to stable storage.

Let $C K T_{i}$ be the checkpointing algorithm associated with $P_{i} . C K T_{i}$ is augmented with the following data structures:

- vol $\log _{i}$ : a volatile log initialized to empty.

- $s n_{i}[1 . . n]:$ an array of sequence number generators. $s n_{i}[j]=\alpha \Leftrightarrow P_{i}$ sent $\alpha$ messages to $P_{j}$.

- known_rec $c_{i}[1 . . n, 1 . . n]$ : a matrix of sequence numbers. known_rec $i[j, k]=\beta \Leftrightarrow P_{i}$ knows $P_{j}$ has received $\beta$ messages from $P_{k}$.

These three data structures are managed ${ }^{6}$ and used by $C K T_{i}$. The following one is managed by $C K T_{i}$ but used (with the previous ones) only by the recovery algorithm (Section 3.4) to compute a set of concurrent local checkpoints.

- $c k p t_{-} t s_{i}[1 . . n]$ : a vector clock for local checkpoints. ckpt_ts $s_{i}[j]=\gamma \Leftrightarrow C K T_{i}$ knows $P_{j}$ has taken $\gamma$ local checkpoints.

\footnotetext{
${ }^{4}$ Remember we are in the context of uncoordinated checkpointing algorithms and, consequently, no additional control messages are used to convey control information.

${ }^{5}$ The most encountered uncoordinated checkpointing algorithm is the periodic one: at regular time intervals, each process individually takes local checkpoints.

${ }^{6}$ If we suppose processes do not send messages to themselves, entries known_reci $[x, x]$ remain equal to zero. These entries can therefore be used to store the array $s n_{i}$ and consequently save space.
}

RR n ${ }^{\circ} 2972$ 
Vector $c k p t \_t s_{i}$ is a vector clock [11] representing $P_{i}$ 's knowledge of the number of local checkpoints taken by each process.

When $P_{i}$ sends or receives a message or when it takes a local checkpoint, $C K T_{i}$ is required to execute the following statements atomically:

when $P_{i}$ sends $m$ to $P_{j}$

begins $s n_{i}[j]:=s n_{i}[j]+1$;

append $\left(m, s n_{i}[j], j\right)$ to vol $\_\log _{i}$;

send $\left(m, k n o w n \_r e c_{i}, c k p t \_s_{i}\right)$ to $P_{j}$

end

when $P_{i}$ receives $\left(m, k \_r, c \_s\right)$ from $P_{j}$

begindeliver $m$ to $P_{j}$;

known_rec $i[i, j]:=k_{\text {known_rec }}[i, j]+1$;

$\forall(x, y):$ known_rec $c_{i}[x, y]:=\max \left(k n o w n \_r e c_{i}[x, y], k \_[x, y]\right)$;

end

$\left.\forall x: c k p t_{-} t s_{i}[x]:=\max \left(c k p t_{-} t s_{i}[x], c\right\lrcorner s[x]\right)$

when a local checkpoint is taken by $P_{i}$

begin $c k p t_{-} t s_{i}[i]:=$ ckpt_t $_{i}[i]+1$;

let $x=$ ckpt_ts $s_{i}[i]$;

let $C_{i}^{x}=P_{i}$ 's current local state; /* last local checkpoint */

$\forall\left(m, s n_{m}\right.$, dest $\left._{m}\right) \in$ vol_log ${ }_{i}:$

do if $s n_{m} \leq k n o w n \_r e c_{i}\left[d_{e s t_{m}}, i\right]$

then suppress $\left(m, s n_{m}\right.$, dest $\left._{m}\right)$ from vol $\log _{i}$

fi

od

save on stable storage $\left(C_{i}^{x}\right.$, vol_log$\left., c k p t \_t s_{i}, k n o w n_{-} r e c_{i}[i, *], s n_{i}\right)$;

$/ *$ known_rec $i[i, *]$ is a vector whose value is the $\mathrm{i}$-th line of the matrix known_rec $c_{i} * /$

vol_log $\log _{i}:=\emptyset$

end

\subsection{A Recovery Algorithm}

A recovery algorithm, similar to the one described in [21], can be associated with the previous checkpointing algorithm. Upon a fault occurrence, the recovery algorithm executes the following steps.

1. First, non-faulty processes are required to take a local checkpoint (this allows as much correct computation as possible to be saved).

2. Then, the most recent set of $n$ concurrent local checkpoints is determined. This determination is done in the following way:

- construct a set $\left\{C_{1}, C_{2}, \ldots, C_{n}\right\}$ by taking the last local checkpoint $C_{i}$ of each process $P_{i}$; let $c_{-} t s_{i}$ be its timestamp (value of $c k p t \_t s_{i}$ when $C_{i}$ is saved on stable storage) ${ }^{7}$.

- while $\exists(i, j): C_{i} \rightarrow C_{j}$

do let $C_{j}^{\prime}$ be the first predecessor of $C_{j}$ such that $\neg\left(C_{i} \rightarrow C_{j}^{\prime}\right)$

$C_{j}:=C_{j}^{\prime}$

od

The set $\left\{C_{1}, \ldots, C_{i}, \ldots, C_{n}\right\}$ obtained contains the latest local local checkpoints which are concurrent.

\footnotetext{
${ }^{7}$ Due to the vector clock properties, we have: $C_{i} \rightarrow C_{j} \Leftrightarrow \forall x: c_{-} t s_{i}[x] \leq c_{-} t s_{j}[x]$ and $c_{-} t s_{i} \neq c_{-} t s_{j}$.
} 


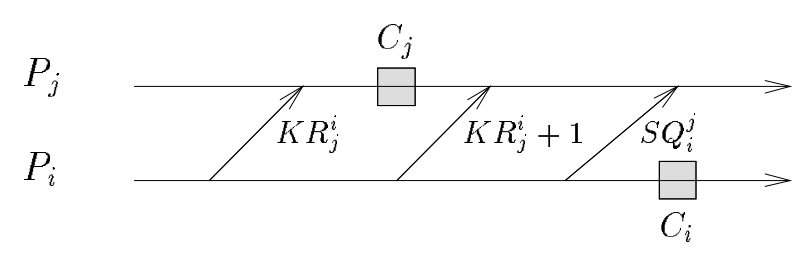

Figure 5: Determining in-transit messages.

3. The state of each channel $c_{i j}$ (messages in transit with respect to the ordered pair $\left(C_{i}, C_{j}\right)$ ) is extracted from the stable storage of $P_{i}$ in the following way. Let $S Q_{i}^{j}$ and $K R_{j}^{i}$ be the values of $s n_{i}[j]$ and known_rec $j[j, i]$ saved on stable storage with $C_{i}$ and $C_{j}$, respectively. As channels are FIFO, messages sent by $P_{i}$ to $P_{j}$ which are in transit with respect to $\left(C_{i}, C_{j}\right)$ have sequence numbers $x$ such that $K R_{j}^{i}<x \leq S Q_{i}^{j}$ (Figure 5).

This set of messages is contained in $P_{i}$ 's stable storage (see the discussion of Section 3.2) from which they can be extracted to constitute a consistent global checkpoint.

\subsection{Discussion}

Unlike coordinated checkpointing algorithms, "pure" uncoordinated checkpointing algorithm cannot a priori prevent the occurrence of the domino effect [13] when a fault occurs. In the worst case, it is not possible to construct a set of $n$ concurrent local checkpoints distinct from the initial set of local checkpoints. To prevent the occurrence of the domino effect, uncoordinated checkpointing algorithms can be made "adaptive": in that case, processes are required to take additional local checkpoints so that any local checkpoint belongs to at least one set of $n$ concurrent local checkpoints. Contrary to coordinated algorithms, the implementation of this "adaptiveness" does not require the addition of control messages. $[1,3,16]$ present such "adaptive" uncoordinated checkpointing algorithms.

Remarks: About strategies for preventing the domino effect.

In [12] Netzer and Xu state and prove a necessary and sufficient condition for a local checkpoint to be useless; a local checkpoint is useless if it cannot belong to any consistent global checkpoint. Useless local checkpoints are the cause of domino effects.

In [4] we formally studied the modeling of consistent global checkpoints and the domino effect in distributed systems. We extended the following result due to Russell [16] (this result states a sufficient condition to eliminate the domino effect).

- Russell's adaptive algorithm. Let $P_{i}$ be a process and $c k p t_{i}$, receive $_{i}$ and $s e n d_{i}$ denote the event " $P_{i}$ takes a local checkpoint", " $P_{i}$ receives a message" and " $P_{i}$ sends a message", respectively. If, for any $P_{i}$, the sequence of events produced by $P_{i}$ obeys the following (regular language) pattern:

$$
\left(\text { ckpt }_{i} \text { receive } e_{i}^{*} \operatorname{send}_{i}^{*}\right)^{*}
$$

then no local checkpoint will be useless, and consequently the domino effect can not occur. It follows that the domino effect will be prevented by forcing each process $P_{i}$ to take an additional local checkpoint between each sequence of send $d_{i}$ events and each sequence of receive $e_{i}$ events.

- Russell's adaptive algorithm follows (in some sense) a "brute force" strategy as the decision to force a process $P_{i}$ to take an additional local checkpoint is only based on $P_{i}$ 's local behaviour.

A more sophisticated algorithm preventing the domino effect is presented in [3]. This adaptive algorithm uses global information (piggybacked by application messages) to force processes to take "as few as possible" additional local checkpoints in order no local checkpoint be useless. When there is no lookahead on the future of the execution (a realistic assumption!) this algorithm is nearly optimal with respect to the number of additional local ckeckpoints that are taken.

End of remarks

RR n ${ }^{\circ} 2972$ 
Local checkpoints and messages logged on stable storage constitute a space overhead. A space reclamation algorithm is described in [22]. Basically, as soon as a consistent global checkpoint has been determined, all local checkpoints and messages that belong to its "past" can be discarded.

Sender-based message logging algorithms have been investigated for a long time [7, 19]. They usually either consider executions of piece-wise deterministic distributed programs $[6,8,19]$ or $\log$ all messages. In the context of receiver-based message logging, [21] characterizes a particular set of messages that are not in transit with respect to any pair of local checkpoints; [23] adapts [21] to the case of sender-based logging. Expressed in our framework, [23] actually considers causal chains composed of only one message; this reduces the size of control information carried by messages but increases the number of messages that are logged on stable storage though they can not belong to a consistent global checkpoint.

A cost of the algorithm we presented lies in the matrix known_rec $c_{i}$, messages have to piggyback. Actually only entries of the matrix corresponding to edges of the communication graph have to be considered. In the case this graph is a directed ring, the matrix shrinks and becomes a vector. Matrices with similar semantics are used (1) in [20] to solve the distributed dictionary problem and (2) in [14] to implement causal message delivery in point-to-point communication networks. As shown in [15] the size of such matrices can be reduced by appropriate techniques when considering the actual communication graph.

\section{Conclusion}

This paper has studied the logging of messages in the context of optimistic sender-based uncoordinated checkpointing algorithms. A global checkpoint is consistent if it is composed of a set of $n$ concurrent local checkpoints and a set of channel states recording all messages sent but not received (in-transit messages) with respect to these local checkpoints.

Coordinated checkpointing algorithms log those messages exactly. Because of their lack of synchronization, uncoordinated algorithms can be more efficient in failure-free computation but conservatively $\log$ more messages. This paper has presented a general technique to reduce the number of messages logged by uncoordinated checkpointing algorithms. An appropriate tracking of the causal dependencies of the underlying computation message exchanges constitutes the core of the protocol.

\section{References}

[1] A. Acharya, B.R. Badrinath, Checkpointing Distributed Applications on Mobile Computers, Proc. 3rd Int. Conf. on Par. and Dist. Information Systems, 1994.

[2] L. Alvisi, K. Marzullo, Message Logging: Pessimistic, Optimistic, and Causal, Proc. 15th IEEE Int. Conf. on Distributed Computing Systems, 1995, pp. 229-236.

[3] R. Baldoni, J. M. Hélary, A. Mostefaoui, M. Raynal, Consistent Checkpointing in Distributed systems, INRIA Research Report 2564, June 1995, 25 p.

[4] R. Baldoni, J. Brzezinski, J.M. Hélary, A. Mostefaoui, M. Raynal, Characterization of Consistent Checkpoints in Large Scale Distributed Systems. Proc. 6th IEEE Int. Workshop on Future Trends of Dist. Comp. Sys., Korea, pp. 314-323, August 1995.

[5] K.M. Chandy, L. Lamport, Distributed Snapshots: Determining Global States of Distributed Systems, ACM Trans. on Comp. Sys., Vol. 3(1), 1985, pp.63-75. 
[6] E.N. Elnozahy, W. Zwaenepoel, Manetho: Transparent Rollback-Recovery with Low Overhead, Limited Rollback and Fast Output Commit, IEEE Trans. on Computers, Vol. 41(5), 1992, pp. 526531.

[7] D.B. Johnson, W. Zwaenepoel, Sender-Based Message Logging, Proc. 17th IEEE Conf. on FaultTolerant Computing Systems, 1987, pp.14-19.

[8] D.B. Johnson, W. Zwaenepoel, Recovery in Distributed Systems Using Optimistic Message Logging and Checkpointing, Journal of Algorithms, Vol. 11(3), 1990, pp.462-491.

[9] R. Koo, S. Toueg, Checkpointing and Rollback-Recovery for Distributed Systems, IEEE Trans. on Software Engineering, Vol. 13(1), 1987, pp. 23-31.

[10] L. Lamport, Time, Clocks and the Ordering of Events in a Distributed System, Communications of the ACM, Vol. 21(7), 1978, pp.558-565.

[11] F. Mattern, Virtual Time and Global States of Distributed Systems. In Cosnard, Quinton, Raynal, and Robert, Editors, Proc. Int. Workshop on Dist. Alg., France, October 1988, pp. 215-226, 1989.

[12] R.H.B. Netzer, J. Xu, Necessary and Sufficient Conditions for Consistent Global Snapshots, IEEE Trans. on Parallel and Distributed Systems, Vol. 6(2), 1995, pp. 165-169.

[13] B. Randell, System Structure for Software Fault-Tolerance, IEEE Trans. on Software Engineering, Vol. 1(2), 1975, pp. 220-232.

[14] M. Raynal, A. Schiper, S. Toueg, The Causal Ordering Abstraction and a Simple Way to Implement it, Inf. Processing Letters, Vol. 39, 1991, pp.343-350.

[15] F. Ruget, Cheaper Matrix Clocks, Proc. 8th Int. Workshop on Distributed Algorithms, Springer Verlag, LNCS 857, pp. 340-354, 1994.

[16] D.L. Russell, State Restoration in Systems of Communicating Processes, IEEE Trans. on Software Engineering, Vol. 6, 1980, pp. 183-194.

[17] L.M. Silva, J.G. Silva, Global Checkpointing for Distributed Programs, Proc. 11th IEEE Symp. on Reliable Distributed Systems, Houston, TX, 1992, pp.155-162.

[18] M. Singhal, F. Mattern, An Optimality Proof for Asynchronous Recovery Algorithms in Distributed Systems, Inf. Processing Letters, Vol. 55, 1995, pp.117-121.

[19] R.E. Strom, S. Yemini, Optimistic Recovery in Distributed Systems, ACM Transactions on Computer Systems, Vol. 3(3), 1985, pp. 204-226.

[20] G.T. Wuu, A.J. Bernstein, Efficient Solutions to the Replicated Log and Dictionary Problems, Proc. 3rd ACM Symp. on Principles of Dist. Comp., 1984, pp. 233-242.

[21] Y.M. Wang, W.K. Fuchs, Optimistic Message Logging for Independent Checkpointing in MessagePassing Systems, Proc. 11th IEEE Symp. Reliable Distributed Systems, 1992, pp. 147-154.

[22] Y.M. Wang, P.Y. Chung, I.J. Lin, W.K. Fuchs, Checkpointing Space Reclamation for Uncoordinated Checkpointing in Message-Passing Systems, IEEE Trans. on Parallel and Distributed Systems, Vol. 6(5), 1995, pp. 546-554.

[23] J. Xu, R.H.B. Netzer, M. Mackey, Sender-Based Message Logging for Reducing Rollback Propagation, Proc. 7th IEEE Symp. on Parallel and Distributed Processing, 1995, pp.602-609, San Antonio.

$\operatorname{RR} n^{\circ} 2972$ 
Unit'e de recherche INRIA Lorraine, Technopôle de Nancy-Brabois, Campus scientifique, 615 rue du Jardin Botanique, BP 101, 54600 VILLERS LÈS NANCY

Unit'e de recherche INRIA Rennes, Irisa, Campus universitaire de Beaulieu, 35042 RENNES Cedex

Unit'e de recherche INRIA Rhône-Alpes, 655, avenue de l'Europe, 38330 MONTBONNOT ST MARTIN

Unit'e de recherche INRIA Rocquencourt, Domaine de Voluceau, Rocquencourt, BP 105, 78153 LE CHESNAY Cedex

Unit'e de recherche INRIA Sophia-Antipolis, 2004 route des Lucioles, BP 93, 06902 SOPHIA-ANTIPOLIS Cedex

\section{Éditeur}

INRIA, Domaine de Voluceau, Rocquencourt, BP 105, 78153 LE CHESNAY Cedex (France)

ISSN 0249-6399 